This is an electronic reprint of the original article. This reprint may differ from the original in pagination and typographic detail.

\author{
Author(s): Johansson, Andreas; Tsai, Hung-Chieh; Aumanen, Jukka; Koivistoinen, Juha; \\ Myllyperkiö, Pasi; Hung, Yi-Zhe; Chuang, Min-Chiang; Chen, Chia-Hao; Woon, Wei Yen; \\ Pettersson, Mika
}

Title: $\quad$ Chemical composition of two-photon oxidized graphene

Year: $\quad 2017$

Version:

Please cite the original version:

Johansson, A., Tsai, H.-C., Aumanen, J., Koivistoinen, J., Myllyperkiö, P., Hung, Y.-Z., Chuang, M.-C., Chen, C.-H., Woon, W. Y., \& Pettersson, M. (2017). Chemical composition of two-photon oxidized graphene. Carbon, 115, 77-82. https://doi.org/10.1016/j.carbon.2016.12.091

All material supplied via JYX is protected by copyright and other intellectual property rights, and duplication or sale of all or part of any of the repository collections is not permitted, except that material may be duplicated by you for your research use or educational purposes in electronic or print form. You must obtain permission for any other use. Electronic or print copies may not be offered, whether for sale or otherwise to anyone who is not an authorised user. 


\section{Accepted Manuscript}

Chemical composition of two-photon oxidized graphene

Andreas Johansson, Hung-Chieh Tsai, Jukka Aumanen, Juha Koivistoinen, Pasi Myllyperkiö, Yi-Zhe Hung, Min-Chiang Chuang, Chia-Hao Chen, Wei Yen Woon, Mika Pettersson

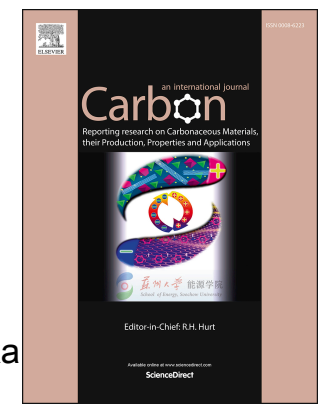

PII: S0008-6223(16)31168-X

DOI: 10.1016/j.carbon.2016.12.091

Reference: CARBON 11602

To appear in: Carbon

Received Date: 11 November 2016

Revised Date: 30 December 2016

Accepted Date: 31 December 2016

Please cite this article as: A. Johansson, H.-C. Tsai, J. Aumanen, J. Koivistoinen, P. Myllyperkiö, Y.Z. Hung, M.-C. Chuang, C.-H. Chen, W.Y. Woon, M. Pettersson, Chemical composition of two-photon oxidized graphene, Carbon (2017), doi: 10.1016/j.carbon.2016.12.091.

This is a PDF file of an unedited manuscript that has been accepted for publication. As a service to our customers we are providing this early version of the manuscript. The manuscript will undergo copyediting, typesetting, and review of the resulting proof before it is published in its final form. Please note that during the production process errors may be discovered which could affect the content, and all legal disclaimers that apply to the journal pertain. 
Graphical abstract

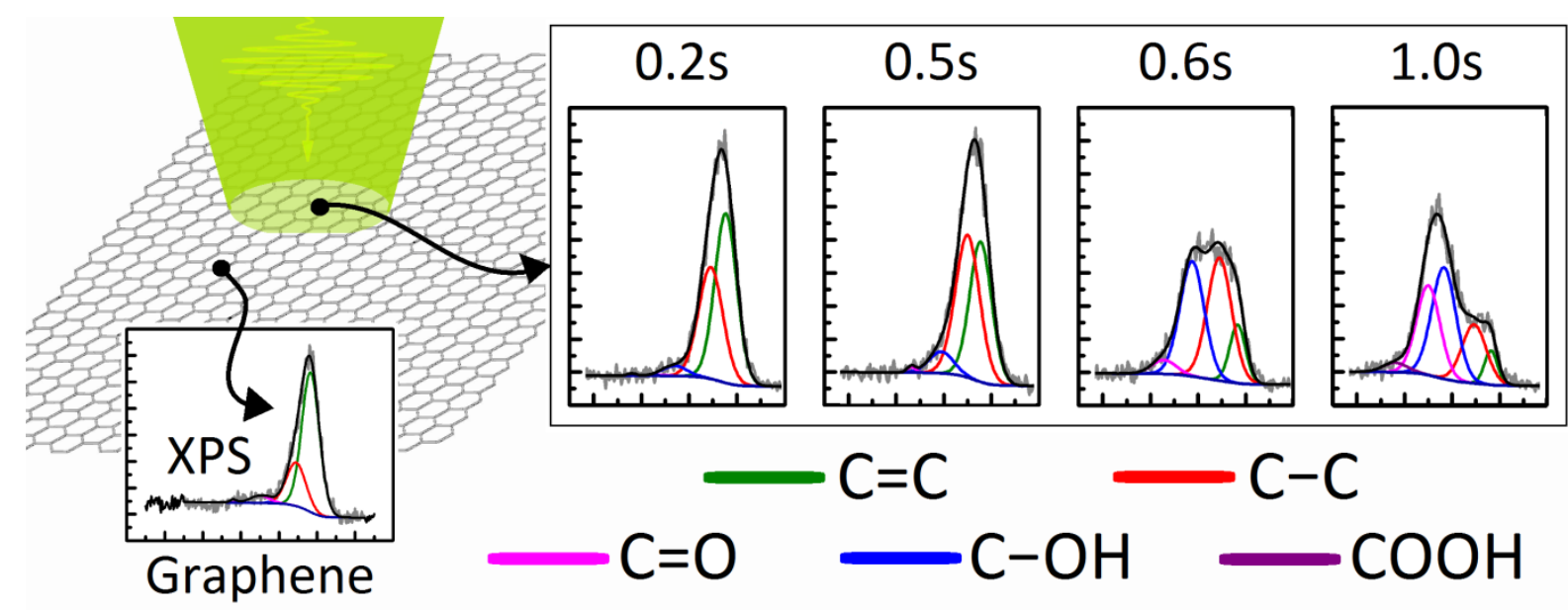




\title{
Chemical composition of two-photon oxidized graphene
}

Andreas Johansson ${ }^{a, b}$, Hung-Chieh Tsai ${ }^{c}$, Jukka Aumanen ${ }^{a}$, Juha Koivistoinen ${ }^{a}$, Pasi Myllyperkiö ${ }^{a}$, Yi-Zhe Hung ${ }^{c}$, Min-Chiang Chuang ${ }^{c}$, Chia-Hao Chen ${ }^{d}$, Wei Yen Woon ${ }^{c^{*}}$, Mika Pettersson ${ }^{a^{*}}$

${ }^{\mathrm{a}}$ Nanoscience Center, Department of Chemistry, P.O. Box 35, FI-40014, University of Jyväskylä, Finland

${ }^{\mathrm{b}}$ Nanoscience Center, Department of Physics, P.O. Box 35, FI-40014, University of Jyväskylä, Finland

${ }^{c}$ Department of Physics, National Central University, Jungli, 32054, Taiwan, Republic of China

${ }^{\mathrm{d} N a t i o n a l ~ S y n c h r o t r o n ~ R a d i a t i o n ~ R e s e a r c h ~ C e n t e r, ~ H s i n c h u, ~ 30076, ~ T a i w a n, ~ R e p u b l i c ~ o f ~}$ China

*Corresponding authors:

Mika Pettersson, E-mail address: mika.j.pettersson@jyu.fi

Wei Yen Woon, E-mail address: wywoon@phy.ncu.edu.tw

\begin{abstract}
Chemical composition of two-photon oxidized single-layer graphene is studied by micrometer X-ray photoelectron spectroscopy (XPS). Oxidized areas with a size of $2 \times 2 \mu \mathrm{m}^{2}$ are patterned on graphene by tightly focused femtosecond pulsed irradiation under air atmosphere. The degree of oxidation is controlled by varying the irradiation time. The samples are characterized by four wave mixing (FWM) imaging and Raman spectroscopy/imaging. Micrometer-XPS is used to study local chemical composition of
\end{abstract}


oxidized areas. XPS imaging shows good contrast between oxidized and non-oxidized areas. Gradual oxidation is observed from growth of signals attributed mainly to hydroxyl (C-OH) and epoxide (C-O-C) groups with a minor contribution of carboxylic groups. The highly oxidized areas are characterized by $\sim 40 \%$ of carbon atoms involved in hydroxyl and $25 \%$ in epoxide groups. The Raman peaks of oxidized graphene are relatively narrow indicating less disordered material than what is typically observed for graphene oxide.

\section{Introduction}

Graphene continues to be a topic of high interest with potential for development of novel applications. ${ }^{1}$ A major goal is to develop transparent, flexible electronics and photonics based on graphene. ${ }^{1,2}$ To this end, new methods are needed for controlled modification of the electronic properties of graphene. Chemical modification is a promising solution for this problem. ${ }^{3}$ Oxidized form of graphene, i.e. graphene oxide (GO) is particularly interesting in this regard since it can be readily made by chemical methods and it improves the solubility and processability of graphene enabling for example making of thin films. ${ }^{4}$ Other useful properties of GO include luminescence and semiconducting or insulating character, depending on the degree of oxidation. ${ }^{4-6}$ Additionally, GO can be reduced thermally or photothermally which can be exploited for device fabrication. ${ }^{7,8}$ Unfortunately, reduction of GO does not lead to pristine graphene but, instead, relatively high degree of oxidation remains in the material reducing electron mobility compared to pristine graphene. ${ }^{9}$ This behavior has been found also in molecular dynamics simulations. ${ }^{10}$ Thus, chemically produced GO is not optimal material for development of graphene based devices.

Recently, two-photon (2P) oxidation of graphene was demonstrated with sub-micrometer

patterning capabilities. ${ }^{11}$ It was shown that by controlling the level of oxidation the local properties of graphene could be tuned. A band gap opening was demonstrated and a field effect transistor (FET) was fabricated with this all-optical method. Oxidation patterning can be conveniently followed by four wave mixing (FWM) imaging. ${ }^{12}$ These developments constitute the basis for direct laser writing of devices on single-layer graphene. In order to enable rational control of the electronic properties of graphene by $2 \mathrm{P}$ oxidation, detailed understanding of the oxidized material is needed. Previous AFM and Raman studies have shown that $2 \mathrm{P}$ oxidation proceeds by generation of point-like functionalized sites which grow into nanosized islands due to the oxidation rate being five orders of magnitude higher for the 
site next to already oxidized site than for the pristine site. ${ }^{13}$ Finally, growing islands coalesce together when their size reaches $\sim 30 \mathrm{~nm}$. This mechanism is very interesting since it leads to two levels of control of the electronic properties of oxidized graphene. Irregular graphene network in between oxidized islands and the width distribution of the graphene channels determines the local band gap, which forms the first level of control. The second level is determined by the level of oxidation inside the islands at the point when they coalesce together forming relatively uniform GO material. Despite the rather detailed picture of the growth mechanism obtained from these studies, there is very limited knowledge on the chemical composition of the $2 \mathrm{P}$ oxidized graphene. Previously it was found that laser irradiation of graphene below ablation threshold produces oxygen-containing groups but more detailed information on the composition and formation dynamics is lacking. ${ }^{14}$

In this work, we study the chemical composition of $2 \mathrm{P}$ oxidized graphene by combining laser fabrication, FWM imaging, Raman spectroscopy and micrometer-XPS. We show that $2 \mathrm{P}$ oxidation leads to formation of $\mathrm{C}-\mathrm{OH}, \mathrm{C}-\mathrm{O}-\mathrm{C}$, and, as a minor species, $\mathrm{COOH}$ groups. Although the formed chemical groups are characteristic of GO, their relative abundance differs from chemically produced GO. Additionally, 2P oxidized graphene is structurally more ordered than chemically produced GO. The results enable rational design of chemical functionalization strategies for the $2 \mathrm{P}$ oxidized graphene which is expected to provide new opportunities for further tuning of the material properties and for sensor development.

\section{Experimental}

2.1 Sample preparation. A silicon chip with $300 \mathrm{~nm} \mathrm{SiO}$ and a monolayer of CVD grown graphene was purchased from Graphenea Inc. Using electron beam lithography and PMMA, a reference grid was patterned, with lines that are $1 \mu \mathrm{m}$ wide and which defines a 10 by 10 matrix of squares, each $200 \mu \mathrm{m}$ by $200 \mu \mathrm{m}$ in size. Reactive ion etching was used to remove graphene from the bottom of the pattern before it was metallized using $2 \mathrm{~nm}$ Ti as adhesion layer and $30 \mathrm{~nm} \mathrm{Au}$ on top. The chip was covered with an additional PMMA layer as protection, and then diced to a suitable size of $5 \mathrm{~mm}$ by $5 \mathrm{~mm}$, before finalizing the patterning with lift-off procedure. The resulting reference grid allows positioning of the $2 \mathrm{P}$ oxidized pattern at a known location so that it can be found during characterization measurements. 
2.2 FWM imaging and non-linear oxidation. Photo-oxidation and FWM imaging of the graphene sample was performed by using the output beams of two non-collinear optical parametric amplifiers (NOPAs, Orpheus-N, Light Conversion) that were pumped with an amplified femtosecond laser (Pharos-10, $600 \mathrm{kHz}$, Light Conversion). Group velocity dispersion of the pulses was pre-compensated by using prism pairs before guiding the beams on the sample through a high numerical aperture microscope objective (Nikon LU Plan ELWD 100x/0.80). The same objective was used for oxidation and FWM imaging. Detailed description of the laser setup can be found from our previous article. ${ }^{11}$

Oxidations were carried out under ambient air using a laser beam of $540 \mathrm{~nm}$ laser pulses with 40 fs pulse duration, $13 \mathrm{pJ}$ pulse energy and $600 \mathrm{kHz}$ repetition rate. Oxidation patterns were formed by moving the sample with a XYZ-piezoscanner in $100 \mathrm{~nm}$ steps to produce $2 \times 2 \mu \mathrm{m}^{2}$ oxidized areas, i.e. each of the squares consists of 400 oxidation spots partially overlapping each other. Oxidation times were $0.1,0.2,0.3,0.4,0.5,0.6,0.8$ and $1.0 \mathrm{~s} / \mathrm{spot}$ and five squares were oxidized with each time parameter forming an $8 \times 5$ matrix of oxidized areas. The separation of the adjacent areas is $2 \mu \mathrm{m}$. After oxidation, graphene was ablated along vertical and horizontal lines next to the oxidized area in order to mark the borders of the processed area. For ablation the pulse energy was increased to $70 \mathrm{pJ}$.

The patterned area was imaged by using FWM imaging. FWM imaging was performed by using two laser beams, with wavelengths $540 \mathrm{~nm}$ and $590 \mathrm{~nm}, 3 \mathrm{pJ} /$ pulse energy and $600 \mathrm{kHz}$ repetition rate. The two beams were adjusted to have spatial and temporal overlap on the sample. FWM signal was collected to backscattering direction and guided to a detector (single photon avalanche photodiode, SPCM-AQRH-14, Excelitas Technologies) by using optical filters. During FWM imaging the sample was kept under nitrogen purge.

2.3 Raman Imaging. Raman measurements were performed with a home-built Raman setup in a backscattering geometry using $532 \mathrm{~nm}$ excitation wavelength produced with $\mathrm{CW}$ single frequency laser (Alphalas, Monolas-532-100-SM). The beam was focused to a sample, and the signal subsequently collected with a 100x microscope objective (Olympus 100x with 0.70 N.A.). The scattered light was dispersed in a $0.5 \mathrm{~m}$ imaging spectrograph (Acton, SpectraPro 2500i) using $600 \mathrm{~g} / \mathrm{mm}$ grating (resolution: $7-8 \mathrm{~cm}^{-1}$ ). The signal was detected with an EMCCD camera (Andor Newton EM DU971N-BV) using $80 \mu \mathrm{m}$ slit width. A beam splitter was placed between the objective and the spectrometer in order to observe the exact measurement point visually. The Rayleigh scattering was attenuated with an edge filter 
(Semrock). The sample positioning was done with a XYZ-piezoscanner (ThorLabs, NanoMax 300). Laser power of $1.5 \mathrm{~mW}$ was utilized and the mapping was conducted using $200 \mathrm{~nm}$ step size and $10 \mathrm{~s}$ accumulation times in a $4 \mu \mathrm{m} \times 20 \mu \mathrm{m}$ area.

2.4 XPS imaging. XPS imaging was performed at the SPEM end station of beamline 09A1 at National Synchrotron Radiation Research Center (NSRRC, Hsinchu, Taiwan). The soft X-ray beam with photon energy of $380 \mathrm{eV}$ was focused with Fresnel zone-plate optics to achieve spatial resolution of $100 \mathrm{~nm}$. The photon energy was calibrated with the core-level line of $\mathrm{Au}$ at binding energy (BE) $84 \mathrm{eV}$. The overall energy resolution is better than $100 \mathrm{meV}$, and the experiments were conducted near $296 \mathrm{~K}$.

\section{Results}

3.1 FWM imaging. A schematic illustration of the arrangement of the irradiated areas and the irradiation times is shown in Figure 1(a) and a FWM image of an oxidized sample is shown in Figure 1(b). The oxidized squares are clearly visible due to the decrease of the FWM signal upon oxidation. The most weakly oxidized squares are hardly visible in the upper part of the Figure. It can also be seen that the trend of increasing oxidation from top to bottom is not entirely monotonous throughout the sample since there are some squares which are more weakly oxidized than the squares above them. This may be due to variation in irradiation conditions during patterning. The bright small spots and lines are due to irregularities in graphene, such as double layers, folds etc. 
(a)

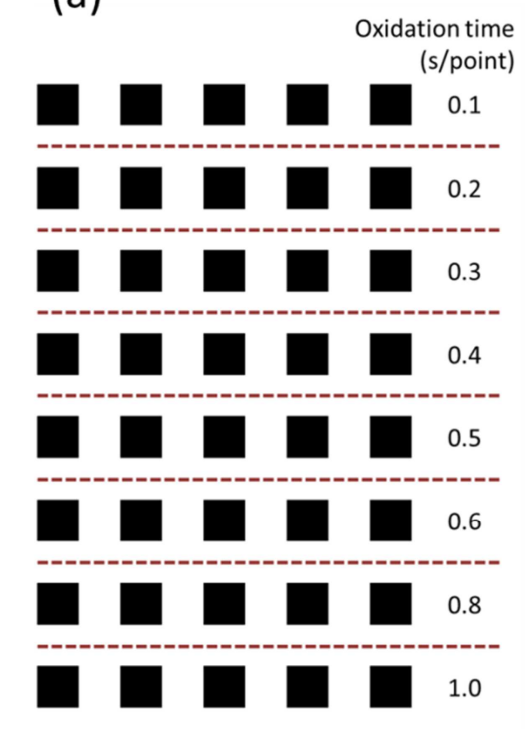

(b)

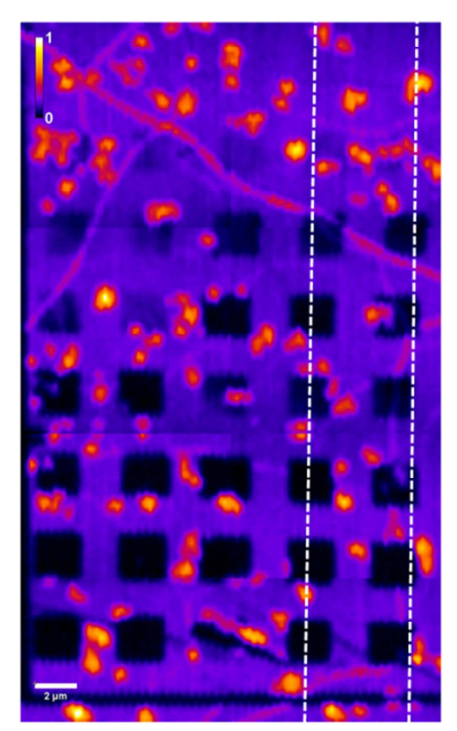

(c)

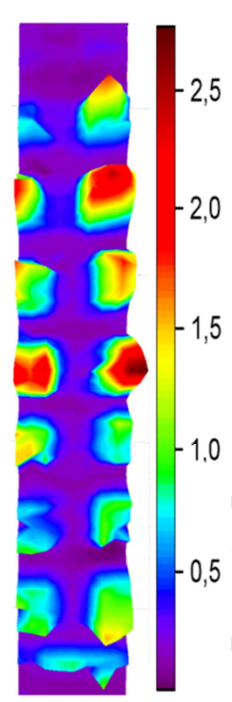

Figure 1. (a) Schematic illustration of the matrix of rectangular areas formed by irradiating graphene with femtosecond laser pulses. Each row has a different irradiation time specified on the right. The five columns are made with the same irradiation parameters. (b) FWM image of a $2 \mathrm{P}$ oxidized graphene sample. The oxidized spots are visible as dark areas due to diminishing FWM signal upon oxidation. (c) Raman image of the area shown in the FWM image (b) by vertical dashed lines. The ratio of the intensities of the $\mathrm{D}$ and $\mathrm{G}$ bands is plotted in the image.

3.2 Raman imaging/spectroscopy. Representative patterned areas were scanned with Raman spectroscopy and an image was constructed representing the ratio of peak heights of the $\mathrm{D}$ and the $G$ band, $I(D) / I(G)$. Raman image of a portion of the sample is shown in Figure 1(c). The oxidized squares are clearly visible in the Raman image due to the growth of the D-band upon oxidation.

Representative Raman spectra from non-irradiated and from irradiated areas of graphene are shown in Figure 2(a). Oxidation is evident by the strong growth of the D-band, broadening and shift of the bands, appearance of the D' band, and decrease of the 2D band. Single Lorentzian functions were fitted to the $2 \mathrm{D}$ and $\mathrm{D}$ bands, and two Lorentzians were used for the $\mathrm{G}$ band in order to take into account the quickly emerging $\mathrm{D}^{\prime}$ band at $\sim 1620 \mathrm{~cm}^{-1}$. In unprocessed graphene, 2D, G and D bands were centered at approximately $2693 \mathrm{~cm}^{-1}, 1593$ 
$\mathrm{cm}^{-1}$ and $1355 \mathrm{~cm}^{-1}$, respectively. The height ratio of the $2 \mathrm{D}$ and $\mathrm{G}$ bands was 1,17 and a single Lorentzian with a width of $29 \mathrm{~cm}^{-1}$ was fitted almost perfectly to the $2 \mathrm{D}$ band, fulfilling the crucial signature of single layer graphene. ${ }^{15}$ For the starting material the Lorentzian fit did not represent the D band equally well as for the oxidized material. However, the height of the D band was captured reasonably well.

Representative Raman spectra of the D, G, and D' bands are shown in Figures 2(b) and 2(c). The $\mathrm{I}(\mathrm{D}) / \mathrm{I}(\mathrm{G})$ ratio as a function of irradiation time is shown in Fig. 2(d). The highest $\mathrm{I}(\mathrm{D}) / \mathrm{I}(\mathrm{G})$ ratio occurs at $0.3 \mathrm{~s}(2,8)$ after which it declines, ending at 1.25 at $1.0 \mathrm{~s}$. At $0.8 \mathrm{~s}$ the intensities of all the bands are decreasing with respect to the silicon peak (of the substrate) at $\sim 1000 \mathrm{~cm}^{-1}$, indicating that the material is partially degrading. Comparison of spectra collected from different oxidation areas showed broadening of all major bands upon oxidation, changing approximately from 29 to $43 \mathrm{~cm}^{-1}, 11$ to $42 \mathrm{~cm}^{-1}$ and 25 to $44 \mathrm{~cm}^{-1}$ for $2 \mathrm{D}, \mathrm{G}$ and $\mathrm{D}$ bands, respectively. The $\mathrm{D}$ band width was determined from the $0.1 \mathrm{~s}$ oxidation spot because in non-irradiated graphene it was too weak.
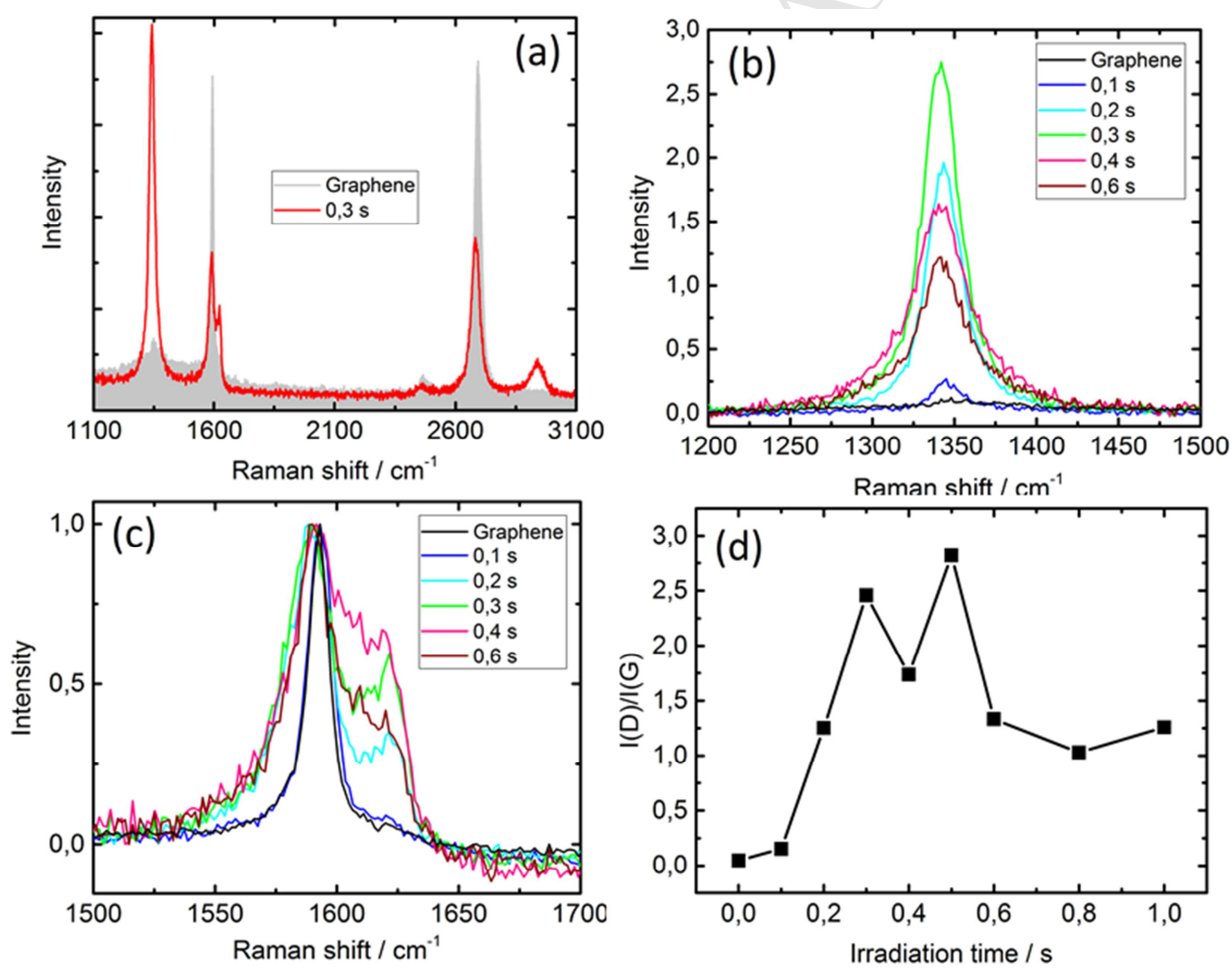

Figure 2. (a) Raman spectra of non-irradiated area of graphene (grey) and area irradiated with $0.3 \mathrm{~s}$ irradiation time (red). (b) D-band at different irradiation times indicated in the Figure. (c) G and D' bands at different irradiation times. The spectra are normalized to the 
maximum of the G-band. (d) Plot of the $\mathrm{I}(\mathrm{D}) / \mathrm{I}(\mathrm{G})$ ratio as a function of irradiation time. The points are average values of $3-5$ individual spectra measured from the center of the irradiated area.

3.3 Micro-XPS. XPS spectra of non-oxidized and oxidized graphene are shown in Figure 3. The spectrum of oxidized graphene is obtained from the heavily oxidized square on the right bottom corner pattern of Fig. 1 (1.0 s/point). The spectra are fitted with multiple mixed Gaussian and Lorentzian functions in order to deconvolute contributions from different chemical groups which have different chemical shifts. The spectra were successfully fitted by using $\mathrm{C}=\mathrm{C}, \mathrm{C}-\mathrm{C}, \mathrm{C}-\mathrm{OH}, \mathrm{C}-\mathrm{O}-\mathrm{C}$ and $\mathrm{COOH}$ groups and their energetic positions are indicated in Fig.3. The main observation is that upon oxidation, several new chemical groups are produced due to a photochemical process and simultaneously carbon belonging to pristine graphene is consumed. Different spectral channels are marked in Fig. 3 with numbers $6-11$ and these areas were later used for imaging and kinetic analysis.

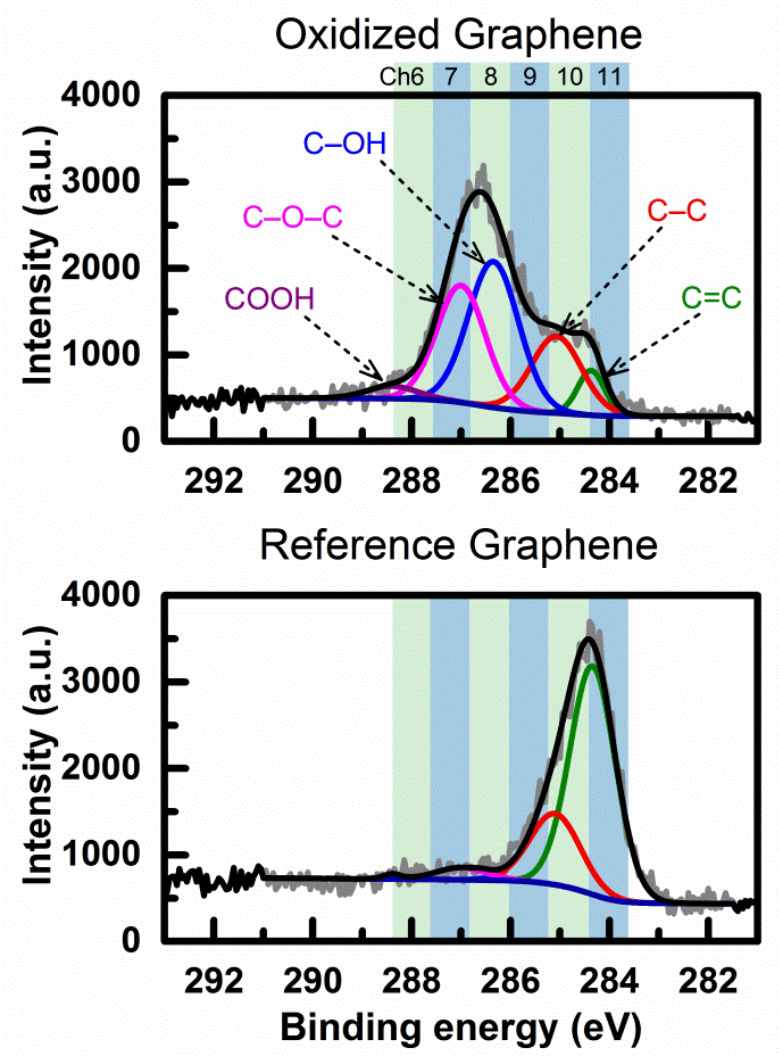

Figure 3. XPS spectra of non-oxidized (below) and oxidized (above) graphene. The fitted spectral components are shown in the Figure. The energies of the components are: $\mathrm{C}=\mathrm{C}$ 
(284.4 eV), C-C (285.0 eV), C-OH (286.4 eV), C-O-C (287.1 eV), COOH (288.6 eV). The channels used in imaging are indicated by colored stripes and the channel numbers are given on top of the upper panel.

An XPS image of the sample is shown in Figure 4(a). The image was generated by plotting the ratio of channels 8 and 11 in order to emphasize the difference produced by irradiation. It is clearly seen that oxidation is localized in the irradiated areas and the image corresponds well with the FWM image in Fig. 1(b). The intensity profile along the vertical line marked by two arrows in Fig. 4(a) is plotted on the right hand side of the XPS image and it shows qualitative correlation with the trend in the irradiation dose, which increases from top to bottom. The evolution of the XPS spectrum as a function of the irradiation dose is presented in Fig. 4(b) along with the fits. The $0.1 \mathrm{~s}$ point is not shown due to small difference to the reference graphene. It is seen that the most dramatic change in the chemical composition takes place between spectra 5 and 6 from the top corresponding to the irradiation times of 0.5 and $0.6 \mathrm{~s}$.
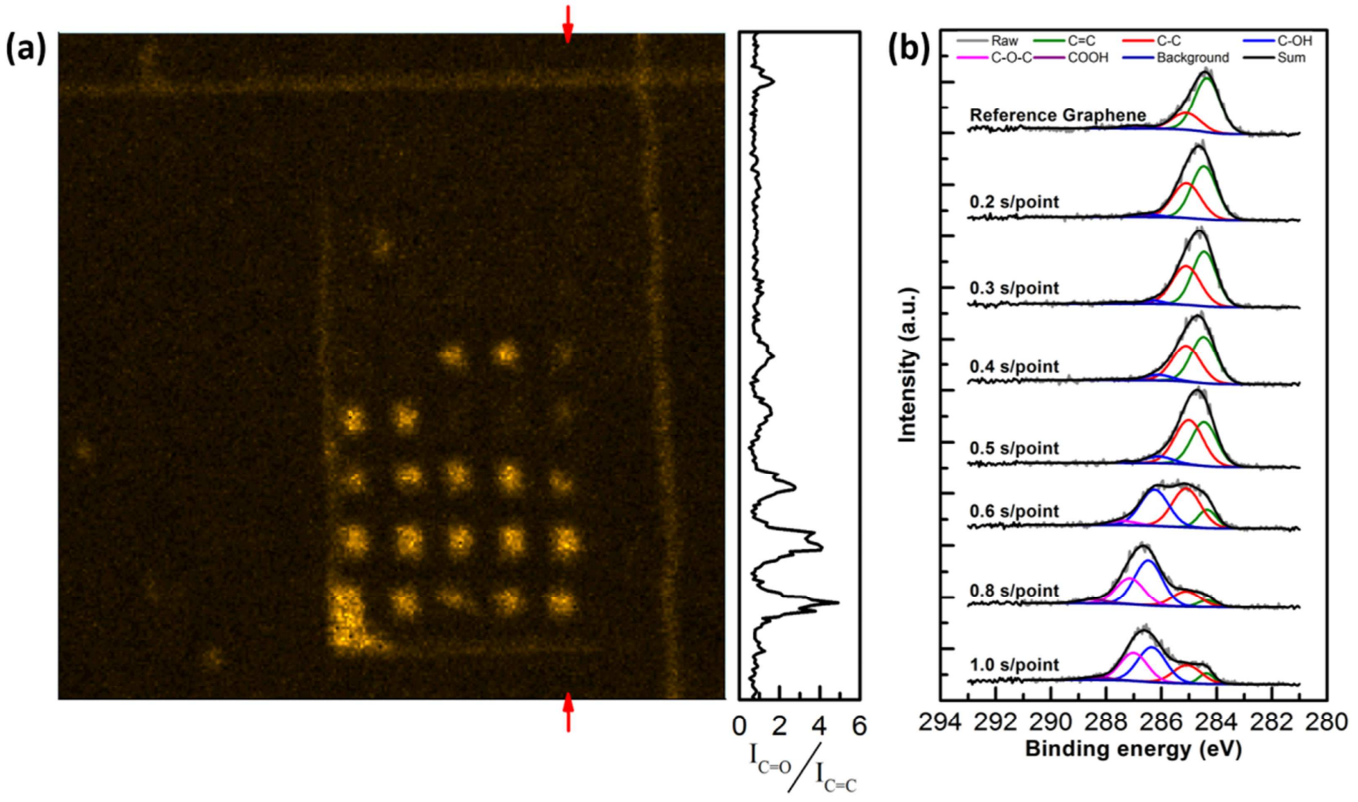

Figure 4. (a) XPS image of the photo-oxidized sample. The ratio of channels 8 and 11 (see Fig. 3) is plotted. The intensity profile along the line shown in the XPS image is shown on the right of the XPS image. (b) XPS spectra from squares irradiated with different doses. 
The evolution of the chemical composition as a function of irradiation time is shown in Figure 5. The plots were generated for each chemical group by taking an average of the fitted amplitude from three columns (1,2 and 4). Between irradiation times of 0.5 and $0.6 \mathrm{~s}$, there is dramatic increase of hydroxyl $(\mathrm{OH})$ and epoxide $(\mathrm{C}-\mathrm{O}-\mathrm{C})$ groups and a decrease of carbon double bonds corresponding to pristine graphene. The rate of change levels off at longer irradiation times and there is no significant change of the $\mathrm{OH}$ group signal between $0.8 \mathrm{~s}$ and $1 \mathrm{~s}$. In the end of the irradiation, about $70 \%$ of carbon atoms are involved in functionalized groups. The proportion of carboxylic groups is highest after $0.8 \mathrm{~s}$ irradiation, being $3.3 \%$ with an error margin of $1 \%$. There is $\sim 1 \%$ of carboxylic acid groups already in the pristine sample.

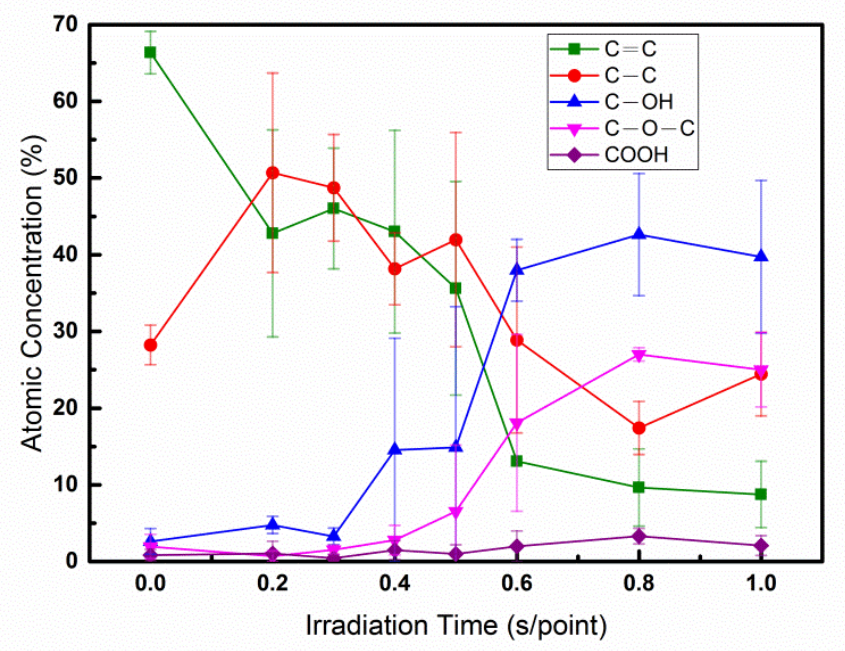

Figure 5. Chemical composition of photo-oxidized graphene analyzed from three columns $(1,2,4)$ of the irradiation pattern (see Fig. 1) by taking an average for each row.

\section{Discussion}

The purpose of the present study is to analyze the chemical composition of $2 \mathrm{P}$ oxidized graphene. The two-photon nature of the oxidation process was previously deduced from the dependence of the FWM signal decay rate on the laser power. ${ }^{11}$ The sample fabrication was designed in such a way that oxidation was localized in well-defined square patterns and the irradiation dose was varied systematically. The pattern was successfully made as seen in the 
FWM image of Fig. 1(b). The Raman image of Fig. 1(c) is compatible with the FWM data. The Raman spectra show increase of the $\mathrm{I}(\mathrm{D}) / \mathrm{I}(\mathrm{G})$ ratio upon irradiation, which can be explained by functionalization of graphene, which changes the hybridization state of carbon from $\mathrm{sp}^{2}$ to $\mathrm{sp}^{3}$. The $\mathrm{I}(\mathrm{D}) / \mathrm{I}(\mathrm{G})$ ratio grows maximally to the value of $\sim 2.8$ for $0.3 \mathrm{~s}$ irradiation time and decreases after that. The peak widths remain narrow only up to the irradiation time of $0.1 \mathrm{~s}$ and broaden after that (see Fig. 2). These observations can be explained by the model of Lucchese et al. which relates the Raman spectral features to the defect density. ${ }^{16}$ By analyzing the The $\mathrm{I}(\mathrm{D}) / \mathrm{I}(\mathrm{G})$ ratio we obtain that the defect density reaches a level of $\sim 10^{13}$ $\mathrm{cm}^{-2}$ for irradiation time of $0.3 \mathrm{~s}$ and after that the density further increases and the material becomes structurally more disordered as evidenced by decreasing $\mathrm{I}(\mathrm{D}) / \mathrm{I}(\mathrm{G})$ ratio and broadening of the Raman peaks. Despite the broadening, the peak widths are remarkably narrow for highly oxidized graphene. The widths remain much narrower compared to chemically oxidized graphene even up to the highest oxidation levels. For example, in Ref. 17, the width of the G-band varied between $45-124 \mathrm{~cm}^{-1}$, depending on the level of oxidation, whereas in our case the maximum width is $42 \mathrm{~cm}^{-1}$. The width of the D-band is maximally $43 \mathrm{~cm}^{-1}$ in our samples whereas the width in chemically produced GO is typically more than $100 \mathrm{~cm}^{-1} \cdot{ }^{4,17}$ Smaller peak width indicates that $2 \mathrm{P}$ oxidation yields less disordered material than chemical oxidation.

The key results in this study are the XPS data. The main observation is that C-OH- and C-OC-groups are formed in 2P-oxidation. The amount of carboxylic groups is very low throughout oxidation. It is not surprising that hydroxyl and epoxide groups are formed since they are typical constituents of graphene oxide. ${ }^{17,18}$ However, the proportion of different chemical groups is different from chemically produced graphene oxide. ${ }^{17}$ In $2 \mathrm{P}$ oxidized graphene the proportion of hydroxyl groups remains larger than the proportion of epoxide groups at all stages while in chemically produced GO, epoxide groups dominate at heavily oxidized material. ${ }^{17}$ In addition, for $2 \mathrm{P}$ oxidized graphene carboxylic groups remain a minor species throughout the oxidation while its amount in chemically produced GO is similar to $\mathrm{OH}$ and epoxide groups. ${ }^{17}$ This may indicate that in $2 \mathrm{P}$ oxidation the carbon network remains unbroken throughout the oxidation since it is widely believed that carboxylic groups are located on the edges of graphene flakes. ${ }^{18,19}$ There is prior evidence that photochemical oxidation of graphene yields structurally good quality material. Mulyana et al. oxidized graphene by UV/ozone treatment which resulted in homogeneous oxidation with $\mathrm{C}-\mathrm{O}-$ 
containing groups. ${ }^{20}$ Oxidized graphene could be reduced back with UV irradiation in the absence of ozone with a good degree of reversibility.

The XPS and Raman observations indicate that not only chemical composition but also structure of $2 \mathrm{P}$ oxidized graphene is different from the chemically produced GO. Thus, $2 \mathrm{P}$ oxidation yields a new composition and structure of GO and this could be important from the point of view of development of applications. In particular, the results indicate that $2 \mathrm{P}$ oxidation has the advantage of tunability of the hydroxyl/epoxide ratio, while still keeping the structural integrity of the material. In contrast, while chemically produced GO allows tuning of the hydroxyl and epoxide ratio in wide range, it usually leads to more disordered structure as indicated by large peak widths in Raman and by the presence of carboxylic groups. Oxidative patterning can also be performed by scanning probe lithography (SPL) but also in that case the broadness of Raman peaks indicates high structural disorder of the resulting material. $^{21}$

Formation of $\mathrm{OH}$ groups indicates that water is involved in the photochemical oxidation mechanism. This is in agreement with several studies that have reported adsorption of oxygen on graphene assisted by water. ${ }^{22-25}$ Additionally, it has been found that water plays a role in photoinduced electrostatic doping of graphene, which also involves oxygen. ${ }^{22-25}$

It is interesting that the main phase of oxidation occurs relatively sharply after a slower initial period. This can be seen in Fig. 5. On the other hand, the Raman data shows rapid increase in the $\mathrm{I}(\mathrm{D}) / \mathrm{I}(\mathrm{G})$ ratio at $0.2 \mathrm{~s}-0.3 \mathrm{~s}$ irradiation time indicating that the main oxidation phase $(0.5-0.6 \mathrm{~s})$ is preceded by a phase during which the defect density reaches $10^{13} \mathrm{~cm}^{-2}$. At this stage the formation of the more disordered phase begins. Further insight and support to this is provided by our previous study where it was found that $2 \mathrm{P}$ oxidation proceeds by growth of nanoislands of oxidized regions until they coalesce together. ${ }^{13}$ The coalescence of islands occurs when their size reaches $\sim 30 \mathrm{~nm}$. Before coalescence, the islands do not progress into highly disordered phase but remain in a state having relatively low "defect" density. It should be noted that "defects" correspond to functionalized (oxidized) sites. The present study shows that after coalescence of islands, corresponding to full consumption of non-oxidized areas of graphene, the degree of oxidation increases rapidly and this explains the XPS observation of strong increase of oxidized groups after initial slow phase. Now, the full picture of the $2 \mathrm{P}$ oxidation mechanism can be formulated: Oxidation starts from point-like functionalizations which occur at sites where oxygen, probably assisted by water, is adsorbed on graphene. 
Initial oxidized areas grow faster than initiation of new sites leading to island-like growth. In this phase, the density of functionalized (i.e. oxidized) sites inside the islands is relatively low. After islands grow large enough $(\sim 30 \mathrm{~nm})$ and coalesce together, the degree of functionalization increases rapidly $(0.5-0.6 \mathrm{~s}$ stage $)$ leading to fast increase in the proportion of mostly hydroxyl and epoxide groups. Final composition with respect to carbon atoms is approximately $40 \% \mathrm{C}-\mathrm{OH}, 25 \% \mathrm{C}-\mathrm{O}-\mathrm{C}$, a few per cent of $\mathrm{COOH}$, and the rest being nonfunctionalized graphene-like material.

The present results are important for understanding the mechanism of $2 \mathrm{P}$ oxidation of graphene and for developing the method further for all-optical device fabrication. Furthermore, identification of the chemical groups present in the oxidized materials enables designing strategies for further functionalization of oxidized graphene. This is particularly interesting from the point of view of developing sensor applications.

\section{Conclusions}

Chemical composition of two-photon oxidized graphene is studied by micro-XPS. Samples containing locally oxidized areas are fabricated with systematic variation of the irradiation dose. XPS analysis shows that functionalization occurs and the main formed groups are hydroxyl $(\mathrm{OH})$ and epoxide (C-O-C) with a few per cent of carboxylic $(\mathrm{COOH})$ groups being observed. The kinetics of functionalization shows sigmoidal behavior with slow initial phase followed by a fast functionalization phase followed by saturation. The fast phase is preceded by reaching a defect (functionalization) density of $\sim 10^{13} \mathrm{~cm}^{-2}$ and coalescence of nanosized oxidized islands, characterized previously. In the final composition roughly $40 \%$ of carbon atoms are involved in hydroxyl groups and $25 \%$ of carbon atoms are involved in epoxide groups. This composition is markedly different from chemically produced graphene oxide, which typically contains higher proportion of epoxide groups and lower proportion of hydroxyl groups for highly oxidized material. The presence of hydroxyl groups indicates that water has an important role in the oxidation mechanism. Remarkably, the G- and D-bands in the Raman spectrum remain relatively narrow up to high oxidation levels indicating structural integrity and modest disorder. The benefits of two-photon oxidation include capability to make patterns and to tune the level of oxidation as well as the hydroxyl to epoxide ratio, while maintaining high structural quality of oxidized graphene. The results are important for the development of electronic devices and sensors from graphene. 


\section{Acknowledgements}

This work is supported by the Ministry of Science and Technology of the Republic of China under contract MOST103-2112-M008-019-MY3. Kevin Roberts is thanked for help in sample preparation.

\section{References}

1. A. C. Ferrari, F. Bonaccorso, V. Falko, K. S. Novoselov, S. Roche, P. Bøggild, et al. Science and technology roadmap for graphene, related two-dimensional crystals, and hybrid systems, Nanoscale 7 (2015) 4598 - 4810.

2. A. D. Franklin, Nanomaterials in transistors: From high-performance to thin film applications, Science 349 (2015) aab2750.

3. E. Bekyarova, M. E. Itkis, P. Ramesh, C. berger, M. Sprinkle, W. de Heer, et al. Chemical modification of epitaxial graphene: Spontaneous grafting of aryl groups, J. Am. Chem. Soc. 131 (2009) $1336-1337$.

4. F. Perrozzi, S. Prezioso, L. Ottaviano, Graphene oxide: From fundamentals to applications, J. Phys. Cond. Mat. 27 (2015) 013002.

5. Z. Luo, P. M. Vora, E. J. Mele, A. T. C. Johnson, J. M. Kikkawa, Photoluminescence and band gap modulation in graphene oxide, Appl. Phys. Lett. 94 (2009) 111909.

6. J.-A. Yan, L. Xian, M. Y. Chou, Structural and electronic properties of graphene oxide, Phys. Rev. Lett. 103 (2009) 086802.

7. Y. Zhang, L. Guo, S. Wei, Y. He, H. Xia, Q. Chen, et al. Direct imprinting of microcircuits on graphene oxides by femtosecond laser reduction, Nano Today 5 (2010) 15 - 20.

8. M. F. El-Kady, R. B. Kaner, Direct laser writing of graphene electronics, ACS Nano 8 (2014) $8725-8729$. 
9. H. A. Becerill, J. Mao, Z. Liu, R. M. Stoltenberg, Z. Bao, Y. Chen, ACS Nano, 2008, 2, $463-470$.

10. A. Bakri, C. Mattevi, M. Acik, Y. J. Chabal, M. Chhowalla, V. B. Shenoy, Structural evolution during the reduction of chemically derived graphene oxide, Nat. Chem. 2 (2010) $581-587$.

11. J. Aumanen, A. Johansson, J. Koivistoinen, P. Myllyperkiö, M. Pettersson, Patterning and tuning of electrical and optical properties of graphene by laser induced two-photon oxidation, Nanoscale 7 (2015) $2851-2855$.

12. J. Koivistoinen, J. Aumanen, V.-M. Hiltunen, P. Myllyperkiö, A. Johansson, M. Pettersson, real-time monitoring of graphene patterning with wide-field four-wave mixing microscopy, Appl. Phys. Lett. 108 (2016) 153112.

13. J. Koivistoinen, L. Sládková, J. Aumanen, P. Koskinen, K. Roberts, A. Johansson, et al. From seeds to islands: Growth of oxidized graphene by two-photon oxidation, J. Phys. Chem. C 120 (2016) 22330 - 22341.

14. I. Bobrinetskiy, A. Emelianov, A. Nasibulin, I. Komarov, N. Otero, P. M. Romero, Photophysical and photochemical effects in ultrafast laser patterning of CVD graphene. J. Phys. D: Appl. Phys. 49 (2016) 41LT01.

15. A. C. Ferrari, Raman spectroscopy of graphene and graphite: Disorder, electron-phonon coupling, doping and nonadiabatic effects, Sol. State. Comm. 143 (2007) 47 - 57.

16. M. M. Lucchese, F. Stavale, E. H. Martins Ferreira, C. Vilani, M. V. O. Moutinho, R. B. Capaz, et al. Quantifying ion-induced defects and Raman relaxation length in graphene, Carbon 48 (2010) 1592 - 1597.

17. K. Krishnamoorthy, M. Veerapandian, K. Yun, S.-J. Kim, The chemical and structural analysis of graphene oxide with different degrees of oxidation. Carbon, 53 (2013) 38 - 49.

18. D. R. Dreyer, S. Park, C. W. Bielawski, R. S. Ruoff, The chemistry of graphene oxide, Chem. Soc. Rev. 39 (2010) 228 - 240.

19. A. Lerf, H. He, M. Forster, J. Klinowski, Structure of graphite oxide revisited, J. Phys. Chem. B, 102 (1998) 4477 - 4482. 
20. Y. Mulyana, M. Uenuma, Y. Ishikawa, Y. Uraoka, Reversible oxidation of graphene through ultraviolet/ozone treatment and its nonthermal reduction through ultraviolet irradiation. J. Phys. Chem. C 118 (2014) 27372 - 27381.

21. H.-C. Tsai, H.-W. Shiu, M.-C. Chuang, C.-H. Chen, C.-Y. Su, J. D. White et al. Graphene reduction dynamics unveiled, 2D Mater. 2 (2015) 031003.

22. N. Mitoma, R. Nouchi, K. Tanigaki, Photo-oxidation of graphene in the presence of water, J. Phys. Chem. C, 117 (2013) 1453 - 1456.

23. S. Ryu, S. Berciaud, Y.-J. Yu, P. Kim, G. W. Flynn,L. E. Brus, Atmospheric Oxygen Binding and hole Doping in Deformed Graphene on a $\mathrm{SiO}_{2}$ Substrate, Nano Lett. 10 (2010) $4944-4951$.

24. Y. Yang, R. Murali, Binding Mechanism of Molecular Oxygen and Moisture to Graphene. Appl. Phys. Lett. 98 (2011) 093116.

25. A. Berholts, T. Kahro, A. Floren, H. Alles, R. Jaaniso, Photo-Activated Oxygen Sensitivity of Graphene at Room Temperature, Appl. Phys. Lett. 105 (2014) 163111. 